\title{
Relaciones entre teoría y práctica en la formación inicial. Percepciones de formadores y estudiantes del Grado de Maestro en Educación primaria
}

\section{Theory-practice relations in initial teacher education. Perceptions of educators and students in the Primary Education Degree}

\author{
Lourdes Montero Mesa ${ }^{1}$ \\ lourdes.montero@usc.es \\ Universidad de Santiago de Compostela, España
}

\section{Resumen:}

El propósito básico de este artículo es el de explorar las relaciones teoría y práctica en la formación inicial del Grado de Maestro en Educación primaria de la Universidad de Santiago de Compostela (USC). El trabajo forma parte de un proyecto de investigación más amplio que se propuso identificar, analizar e interpretar las percepciones de futuros profesores y sus formadores sobre los conocimientos que están construyendo en el desarrollo del Plan de estudios del Grado de Maestro en Educación Primaria y la contribución de estos a configurar una identidad profesional determinada. Con un enfoque metodológico cualitativo se utilizó análisis documental, relatos, grupos de discusión y entrevistas a profesorado, coordinadores de titulación, decanos, estudiantes y tutores/as de Practicum durante el periodo 2013/2016. Para su análisis se ha emplea-

1 Dirección para correspondencia (correspondence address):

Lourdes Montero Mesa. Dpto. de Pedagoxía e Didáctica. Universidad de Santiago de Compotela. Facultade de Ciencias da Educación. Campus Vida. 15782 Santiago de Compostela (España). 
Relaciones entre teoría y práctica en la formación inicial. Percepciones de formadores y estudiantes del Grado de Maestro en Educación primaria

Lourdes Montero Mesa

do una categorización deductiva-inductiva llevada a cabo con el apoyo del soporte informático Atlas.ti.

El análisis e interpretación de los datos evidencia un modelo de separación teoría y práctica en el que la teoría precede a la práctica y ésta es comprendida como aplicación de la teoría. Una situación que genera tensiones e insatisfacción entre formadores -profesores y tutores- y estudiantes, pero también la búsqueda de estrategias alternativas para la co-implicación deseable entre ambos tipos de saberes. Sin embargo, los obstáculos son más poderosos que los deseos de cambiar una realidad socialmente construida en la que el juego dialéctico, bidireccional, se constituya en principio para la acción.

\section{Palabras clave:}

Relación teoría y práctica; conocimiento profesional; formación inicial del profesorado; maestros educación primaria. theory and practice in which theory precedes practice and this is understood as an application of theory. This is a situation that generates tensions and dissatisfaction between educators -teachers and tutors- and students, but also the search for alternative strategies for the desirable co-implication between both types of knowledge. However, the obstacles are more powerful than the desire to change a socially constructed reality in which the two-way dialectical game becomes a principle for action.

\section{Key words:}

Theory and practice relationship; professional knowledge; initial teacher education; primary school teachers.

\section{Résumé:}

Le but fondamental de cet article est d'explorer les relations théoriques et pratiques dans la formation initiale du Professeur en Enseignement Primaire à l'Université de Saint Jacques de Compostelle (USC). Le travail fait partie d'un projet de recherche plus large visant à identifier, analyser et interpréter les perceptions des futurs enseignants et de leurs formateurs sur les connaissances qu'ils construisent dans le développement du programme de formation initiale en éducation primaire et la contribution de ceux-ci pour configurer une certaine identité professionnelle. Grâce à une approche méthodologique qualitative, des analyses documentaires, des histoires, des groupes de discussion et des entretiens avec des enseignants, des coordinnateurs de diplômes, des doyens, des étudiants et des tuteurs de stage au cours de la période 2013-2015 ont été utilisés. Pour leur analyse, une catégorisation déductive-inductive a été réalisée avec le logiciel Atlas.ti. L'analyse et l'interprétation des données mettent en évidence un modèle théorique et pratique séparés, dans lequel la théorie précède la pratique, qui est comprise comme une application de la théorie. Une situation qui génère des tensions et des insatisfactions entre les formateurs -enseignants et tuteurs- et les étudiants, mais aussi la recherche de stratégies alternatives pour la co-implication souhaitable entre les deux types de connaissances. Cependant, les obstacles sont plus puissants que le désir de changer une réalité socialement construite dans laquelle le jeu dialectique bidirectionnel devient un principe d'action.

\section{Mots clés:}

Relation théorie et pratique; connaissance professionnelle; formation initiale des enseignants; professeur d'école primaire.

Fecha de recepción: 10-2-2018

Fecha de aceptación: 14-5-2018 


\section{Introducción}

Las relaciones entre la teoría y la práctica se presentan en la formación del profesorado como una de esas cuestiones perdurables que no se resuelven de una vez por todas. Atender la complejidad de esta relación desborda los límites de este artículo focalizado en la formación inicial. En esta etapa, la entendemos como un paraguas conceptual que cubre las interacciones entre las materias de los planes de estudio, las actuaciones de formadores y estudiantes y las experiencias de campo en el interior de las instituciones de formación inicial; pero también, las relaciones entre las universidades y centros educativos, entre estos y la administración educativa, las políticas, las prácticas y la investigación.

El trabajo forma parte de un proyecto de investigación coordinado llevado a cabo por los grupos de investigación Stellae (http://stellae.usc. es) y FODIP (http://www.ub.edu/fodip/) de las Universidades de Santiago de Compostela (USC) y Barcelona (UB), financiado por el Ministerio de Economía y Competitividad (MINECO), titulado "Desarrollo del conocimiento profesional a través del plan de estudios del Grado de Maestro en Educación primaria. Perspectivas del alumnado y el profesorado". En este artículo exploramos la percepción de formadores -profesores de la universidad y tutores de practicum- y estudiantes de la USC sobre las relaciones entre la teoría y la práctica en la construcción del conocimiento profesional durante la formación inicial.

La relación entre el conocimiento teórico y la práctica profesional continúa siendo un tema endémico en la formación del profesorado. El debate permanece abierto y tiene la suficiente complejidad epistemológica y política como para no aspirar a dar un retrato omnicomprensivo o darlo por zanjado (Álvarez Álvarez, 2011; Escudero, 1993; Gimeno, 1998; Medina Moya, 2017; Pérez Gómez, 1993; Tardiff, 2004; Zeichner, 2010).

El artículo está estructurado en cuatro grandes apartados: un marco teórico, marco empírico con la referencia a la metodología empleada, resultados y discusión y conclusiones. 
Relaciones entre teoría y práctica en la formación inicial. Percepciones de formadores y estudiantes del Grado de Maestro en Educación primaria

Lourdes Montero Mesa

\section{Marco teórico}

Tres apartados configuran el marco teórico: Pertinencia y relevancia de la formación inicial; significados de la relación teoría y práctica. Breve referencia al practicum.

\subsection{De la pertinencia y relevancia de la formación inicial}

La formación del profesorado, es uno de los ámbitos que mejor refleja la complejidad de los cambios en educación y su relación con dinámicas sociales, económicas, políticas y tecnológicas más amplias, lo que en ocasiones hace de él un asunto político prioritario. Suele ocurrir cuando evaluaciones de la calidad del sistema educativo iluminan los problemas y la formación del profesorado se contempla como la mejor solución, olvidando que es, a su vez, el problema más grave en educación, como afirma Fullan (2002). Cuando los ecos de esas evaluaciones se van extinguiendo, se apaga también la euforia reformista; cuáles fueron los cambios y transformaciones producidas, cuales no y por qué, cuál es la percepción de sus protagonistas, permanecen como cuestiones de interés para la investigación (Escudero, 1993, 2006; Gewerc y AlonsoFerreiro 2017; Rodríguez Romero, 2001).

La revisión de Cochran-Smith y Villegas (2016) sobre el estado de la investigación sobre la formación inicial del profesorado -preparación e inducción- pone el foco sobre estas cuestiones dibujando un paisaje claramente impactado por las condiciones sociales, económicas, políticas y tecnológicas de la sociedad global del conocimiento con tres argumentos principales. El primero, plantea un marco teórico que conceptualiza la investigación sobre la formación inicial como una práctica social históricamente situada, donde concurren investigadores con diferentes enfoques y objetivos. Identifican tres grandes programas de investigación en las últimas décadas, representativos de tres grandes tendencias ideológicas en competición: 1) Rendición de cuentas (accountability) y calidad docente, revela las tendencias políticas en la formación inicial y concita una atención sin precedentes; 2) la preparación del profesorado para la sociedad del conocimiento, muestra las tendencias sobre las concepciones cambiantes de cómo aprenden y qué necesitan conocer las personas en una economía del conocimiento, y 3) la preparación del profesorado para la diversidad y la equidad refleja las tendencias demo- 
gráficas del incremento de la diversidad de las poblaciones estudiantiles y la desigualdad escolar creciente.

El segundo, defiende las relaciones existentes entre las prácticas de investigación, por un lado, y el poder social, político, económico e institucional, por otro. Para las autoras, hay diferencias en el grado en el que las investigaciones se alinean con la agenda de reforma neoliberal dominante.

El tercero, alude a las limitaciones de la revisión y propone nuevas líneas de investigación:

1) Ampliar y profundizar la comprensión de la práctica de la formación y el aprendizaje de los candidatos más allá de un curso, experiencias de campo y programa, atendidos en los estudios revisados.

2) Examinar en el tiempo cómo y bajo qué condiciones los futuros profesores aprenden las complejas habilidades de la enseñanza como prácticos reflexivos, a negociar habilidades y prácticas clave y adaptarlas a las necesidades de los estudiantes.

3) Explorar cuestiones desarrolladas conjuntamente por la escuela y la universidad sobre las conexiones entre el aprendizaje de los profesores y el de los estudiantes, incluyendo las condiciones que apoyan u obstaculizan la construcción de entornos para enriquecer las oportunidades de estudiantes marginados.

4) Reclamar más investigación sobre las relaciones de la formación del profesorado y los factores sociales, culturales e institucionales, en particular el impacto de la pobreza sobre la enseñanza, el aprendizaje y la formación del profesorado.

Esta breve referencia a la revisión de Cochran-Smith y Villegas (2016) -1500 estudios en el periodo 2000-2012- destaca la enorme importancia de la investigación y subraya la relevancia de la formación inicial, no siempre reconocida en nuestro contexto (Bolívar, 2006; Montero, 2008). Los tres programas y tendencias de investigación identificados, constituyen un marco de referencia para el objetivo de este artículo por entender que la formación inicial ha sido históricamente un buen ejemplo para estudiar las relaciones entre teoría y práctica. Por un lado, al suscitar contundentes declaraciones sobre su relevancia junto a condiciones que desafían con la misma contundencia esas declaraciones.

La formación inicial del profesorado de Infantil, Primaria y Secundaria se ha visto afectada por los cambios producidos en el sistema edu- 
Relaciones entre teoría y práctica en la formación inicial. Percepciones de formadores y estudiantes del Grado de Maestro en Educación primaria

Lourdes Montero Mesa

cativo español en las últimas décadas. El más decisivo, la reforma emprendida en 2007 como consecuencia del desarrollo de la Ley Orgánica de Educación (LOE, abril 2006) y su adaptación a las previsiones de la Ley Orgánica de Universidades (LOU, mayo 2007). La transformación principal consistió en la sustitución del modelo establecido por la Ley General de Educación y Financiamiento de la Reforma Educativa (LEGE, agosto 1970) por el actual modelo basado en el sistema de grados y postgrados del Espacio Europeo de Educación Superior (EEES) (Tiana, 2013).

Las reformas en la formación inicial son incompletas e inacabadas, al vaivén pendular de los cambios sociales, políticos y económicos. Cambios que se reflejan en tres elementos: (i) las competencias necesarias para ejercer la docencia que terminan por configurar y definir los planes formativos; (ii) el estatuto epistemológico y académico de la formación; y (iii) el modelo formativo, con especial énfasis en la relación entre la teoría y la práctica, cuyas combinaciones generan modelos de formación inicial distintos (Prats, 2016).

Uno de los cambios más relevantes producidos fue el salto de la diplomatura al grado que representa el ascenso de otro escalón en la jerarquía universitaria: de ser una titulación de primer ciclo a las de segundo y posibilitar acceso al tercero. Una reivindicación históricamente mantenida, truncada en las promesas incumplidas por la Ley Orgánica de Ordenación General del Sistema Educativo (LOGSE, octubre 1990), que mantuvo el estatus quo de la formación inicial (a pesar del desafío producido por la reconfiguración de la etapa educativa obligatoria), priorizando la formación del profesorado en ejercicio.

El cambio, largamente reivindicado, se produjo gracias al proceso de armonización en un EEES, como afirma uno de los profesores de nuestro estudio:

... nos vino dado directamente por el EEES, sin tener que pedirlo, de tal manera que cuando todo el mundo protesta por el Plan Bolonia yo digo no, no, nosotros no porque él solito nos ha dado lo que por otros cauces y por muchas argumentaciones y justificaciones nunca habíamos podido lograr, ni hubiéramos logrado si no fuera por esta circunstancia porque si para nosotros institucionalmente fue una salvación para el estudiantado fue mucho más porque nos vino a homologar directamente con el resto de los estudiantes universitarios (Entr.Prof.1.Lugo). 
La investigación que alimenta este artículo se sitúa en este contexto de cambio pues está realizada en el marco del plan de estudios derivado de la armonización en un EEES (2010-2014), en la USC, en las facultades de los campus de Santiago y Lugo. En ambas, los 240 créditos a cursar por el alumnado se distribuyen en cuatro cursos de 60 créditos cada uno, en asignaturas con carácter cuatrimestral, a excepción del Trabajo Fin de Grado (TFG). Aumentar los créditos del practicum ha sido uno de los resultados de la implantación del grado frente a la diplomatura. En el caso de la USC ha supuesto la organización de sus 42 créditos totales en tres cursos $\left(2^{\circ}, 3^{\circ}\right.$, y $\left.4^{\circ}\right)$.

\subsection{De los significados de la relación teoría y práctica}

Las relaciones entre teoría y práctica constituyen un problema persistente en el proceso de construcción del conocimiento profesional docente. La red que entrama ambas cuestiones es densa, cuesta desentrañar su naturaleza y atribuir mayores o menores dosis de responsabilidad en la mejora de la formación del profesorado (Montero, 2001, 2006, 2017).

El problema de la relación entre la teoría y la práctica transciende el ámbito de la actividad profesional de la enseñanza y la formación del profesorado a otros ámbitos de la educación y de la construcción de conocimiento en general. El primer problema es saber qué entendemos por teoría y por práctica:

...práctica y teoría son, dos comodines del lenguaje, cuya significación dispersa complica bastante la manera de entender la relación entre ambos. Esta falta de precisión hace de la relación teoría-práctica un eslogan, una metáfora, una aspiración, un programa difuso, un arma arrojadiza para descalificarse entre unos y otros, para poner en cuestión o para afianzar una determinada división del trabajo. (Gimeno, 1998, p. 33).

Entiendo por teoría, el conjunto de conocimientos -conceptos, creencias, habilidades, disposiciones- y compromisos vehiculados por las diferentes materias del currículo de la formación inicial del profesorado, con la expectativa de conformar el bagaje intelectual y emocional necesario para afrontar la actividad profesional de la enseñanza por los futuros maestros. Saberes constituidos (Contreras, 2013). Entiendo por práctica el conjunto de oportunidades de observación, análisis e inter- 
Relaciones entre teoría y práctica en la formación inicial. Percepciones de formadores y estudiantes del Grado de Maestro en Educación primaria

Lourdes Montero Mesa

pretación del papel de profesor en situaciones de enseñanza, simuladas, virtuales o reales. Los distintos tipos de prácticas que tienen lugar en las instituciones de formación inicial no son la práctica profesional sino ocasiones para aprender ésta.

Los significados que conferimos al término teoría están en función de las estructuras de racionalidad predominantes en cada campo de conocimiento. La concepción de la relación teoría y práctica que otorga a la primera superioridad para guiar la segunda representa la hegemonía de la racionalidad técnica en la construcción del conocimiento y en la formación de profesionales (Carr y Kemmis, 1988; Gimeno, 1998; Medina Moya, 2017; Pérez Gómez, 1993; Schön, 1992). La supremacía de la teoría sobre la práctica se pone de manifiesto en la dicotomía teoría y práctica, teóricos (investigadores, formadores) y prácticos (profesores). Los unos construyen conocimiento y de los otros se espera que lo apliquen en sus aulas. El desencuentro entre ambos puede explicarse en las diferencias culturales que dificultan la comunicación. Para Medina Moya (2017, p. 34) reside en la necesidad de establecer la conexión entre las "estructuras teóricas generadas por la investigación y los esquemas de interpretación de los docentes".

La relación teoría y práctica se ha venido caracterizando por un juego de presiones entre la distancia que las separa y la aspiración de su interacción (Montero 2001, 2006, 2017). Potenciar la relación entre la teoría y la práctica requiere concebirla desde una dimensión reflexiva en la medida en ésta implica un fuerte desarrollo conceptual sobre la naturaleza del trabajo profesional del profesorado, las interacciones en las instituciones de formación y los roles a desempeñar por formadores, estudiantes y otros profesionales en los procesos de enseñanza y aprendizaje de la profesión.

\subsubsection{De LA SEPARACIÓN ENTRE TEORÍA Y PRÁCTICA}

El modelo dominante de la formación inicial del profesorado,

...es el que responde a la teoría implícita de la integración inmediata: la universidad proporciona las teorías, métodos y habilidades; las escuelas proporcionan aulas, curriculum, alumnos; y el futuro profesor proporciona el esfuerzo individual; todo lo cual se combina para producir un resultado acabado de un profesor profesional. Este modelo de formación ignora, sin embargo, el contexto social y político de la formación, mientras subraya el esfuerzo individual... (Britzman, 1986, p. 442). 
Contemplar de modo segmentado las relaciones entre teoría y práctica otorga a la primera la misión de anticipar la segunda. Este esquema propio de la racionalidad técnica, trae como consecuencia la dicotomía entre ambas de manera tal que acaban siendo dos realidades distantes una de la otra. El conocimiento teórico, formal, es el más relevante.

La teoría tiene una capacidad constructiva limitada: "esperar que la práctica pueda ser deducida de la teoría y gobernada por ella es una creencia equivocada (...) la teoría tiene fundamentalmente una función crítica y reflexiva" (Veenman, 1984, p. 167). El objetivo de la teoría no es alentar la conformidad con una determinada tradición o unas determinadas prescripciones científicas, sino posibilitar

...una disposición para hacer juicios profesionales... La teoría se relaciona con la práctica ilustrando a los practicantes; aspira a educar a los practicantes, a profundizar sus puntos de vista y animar sus compromisos para que puedan ver más profundamente bajo la superficie de sus ideas y de sus prácticas. (Carr, 1990, p. 81).

En el contexto de la formación inicial, la relación entre teoría y práctica suele limitarse a las experiencias de campo, al modelo de sucesión teoría y práctica que continúa vigente en la percepción de estudiantes y formadores. Para Carr (1990, p. 52):

...las distancias entre la teoría y la práctica están totalmente inmersas en los fundamentos conceptuales sobre los que se ha construido el conjunto de la práctica sólo (...) se eliminarán retirando algunos supuestos básicos dudosos en cuyos términos se ha entendido siempre la teoría y su relación con la práctica.

Quienes han alimentado una posición de alejamiento profesional sistemático e institucionalizado de la práctica de la enseñanza, lo han hecho desde creencias y valores que tienen que ver o bien con una infravaloración de la práctica, o bien con un cierto mantenimiento de la distancia por su bajo estatus, o con ambas razones a la vez (Hargreaves, 1999; Labarée, 1999). Sea como fuere, es innegable el hecho de que la formación del profesorado opera en dos marcos de actuación difíciles de reconciliar: el de los mundos virtuales y el de los mundos reales de la enseñanza, cual dios Jano. 
Relaciones entre teoría y práctica en la formación inicial. Percepciones de formadores y estudiantes del Grado de Maestro en Educación primaria

Lourdes Montero Mesa

\subsubsection{De LA INTERRELACIÓN ENTRE TEORÍA Y PRÁCTICA}

Reducir distancias entre la teoría y la práctica es el objetivo central de la teoría educativa (Carr y Kemmmis, 1988, p. 129). Conseguirlo implica mejorar la 'eficacia práctica' de las teorías de los profesores en lugar de mejorar la eficacia práctica de los productos de las actividades teóricas.

Una de las razones aducidas para explicar la persistente distancia entre teoría y práctica aludía al hecho de que los teóricos de la enseñanza y la formación del profesorado con frecuencia construyen sus modelos de competencia sin considerar las teorías implícitas del profesorado. En el territorio de los prácticos, no importa quién haya conceptualizado qué, ni desde dónde y con qué propósitos o bajo qué contextos de aplicación lo ha pensado útil y relevante. Más bien se trata de 'probar y contrastar' lo que se ofrece desde la teoría. Y cuando la cuestión consiste en 'probar y contrastar', los referentes mejor aceptados no son los teóricos sino los propios prácticos, o aquellos que lo han sido y han pasado a ocupar un espacio intermedio entre la teoría y la práctica. En general, todos aquellos agentes de formación que mantienen o han mantenido una relación directa con las aulas.

Sin embargo, no es suficiente con esta mediación de los agentes teórico-prácticos de la formación. La propia teoría de la enseñanza está implícita en la práctica de la misma. Y esto proporciona un valor singular al término teoría que puede ayudar a construir puentes entre las teorías de los teóricos y las prácticas de los prácticos. Nos referimos al conocimiento práctico en sus tipos, componentes y modos de acceso (Colén, 2017; Ben-Perezt, 2011; Montero, 2001, 2016):

...esto no significa que la relación entre lo teórico y lo práctico sea tal que la teoría 'implique' la práctica, ni que se 'derive' de la práctica, ni siquiera que 'refleje' la práctica. Se trata de que, al someter a una reconsideración racional las creencias y justificaciones de las tradiciones existentes y en uso, la teoría informe y transforme la práctica, al informar y transformar las maneras en que la práctica se experimenta y entiende. Es decir que no hay transición de la teoría a la práctica como tal, sino más bien de lo irracional a lo racional, de la ignorancia y el hábito al conocimiento y a la reflexión. (Carr y Kemmis, 1988, p. 128).

El conjunto de teorías explícitas e implícitas que los profesores ponen en juego en la acción docente implican un conocimiento profesional 
que, de algún modo, les convierte también en teóricos. Los profesores transforman inevitablemente el contenido de la enseñanza en algo que tiene su propia lógica a la búsqueda del sentido para el alumnado.

Este trabajo didáctico de transformación viene a ser una manifestación posible del puente de unión entre teoría y práctica. Tender puentes de contenido entre los conocimientos teóricos de origen y los conocimientos prácticos del aula (Zeichner, 2010). Un diálogo imprescindible para comprender a los profesores como investigadores de su propia práctica y la indagación como enfoque de la formación del profesorado (Elliott, 2010; Lieberman y Miller, 2003). De la concepción de los profesores como "consumidores de conocimiento" a la de "productores" (Escudero y Luis, 2006).

\subsection{Del practicum}

Se entiende que la experiencia directa de los estudiantes con sus futuros escenarios laborales es el puente por excelencia para las expectativas y oportunidades de relación entre la teoría y la práctica. Y es por ello que el practicum suscita un acuerdo casi generalizado sobre su valor por parte de los futuros profesores. La abundante investigación disponible constata que continúa siendo el componente curricular de la formación inicial más valorado pero también examinado y criticado (White y Forgasz, 2016).

La condición del practicum como experiencia directa de la realidad no impide recordar tres cosas. La primera, que en la formación inicial del profesorado tienen cabida otro tipo de prácticas, de experiencia indirecta, proporcionadas por estrategias metodológicas como estudios de caso, simulaciones, grabaciones (laboratory experiences), accesibles con las tecnologías existentes. La segunda, que a partir de la reforma de los planes de estudio en la universidad, todas las materias se articulan con créditos teóricos y prácticos lo que posibilita un incremento de la experiencia indirecta y la reflexión sobre la misma. La tercera propuesta por Schön:

Un practicum es una situación pensada y dispuesta para la tarea de aprender una práctica. En un contexto que se aproxima al mundo de la práctica, los estudiantes aprenden haciendo, aunque su hacer a menudo se quede corto en relación con el trabajo propio del mundo real. Aprender haciéndose 
Relaciones entre teoría y práctica en la formación inicial. Percepciones de formadores y estudiantes del Grado de Maestro en Educación primaria

Lourdes Montero Mesa

cargo de proyectos que simulan y simplifican la práctica, o llevarlos a cabo, relativamente libres de presiones, las distracciones y los riesgos que se dan en el mundo real al que, no obstante, el practicum hace referencia. Se sitúa en una posición intermedia entre el mundo de la práctica, el mundo de la vida ordinaria, y el mundo esotérico de la universidad. (Schön, 1992, p. 46).

El practicum no es la práctica, pero representa sin duda una extraordinaria oportunidad para aprenderla, de dónde el empeño por considerarlo como el 'corazón' de los programas de formación inicial del profesorado (Ferreres, 1998; White y Forgasz, 2016; Zeichner, 2010).

\section{Marco empírico}

Anticipaba en la introducción la referencia al desarrollo entre 20132016 de un proyecto de investigación coordinado titulado "Desarrollo del conocimiento profesional a través del plan de estudios del Grado de Maestro en Educación primaria. Perspectivas del alumnado y el profesorado", financiado por el MINECO (Referencia EDU2012-39866-C02-01). El estudio se propuso analizar e interpretar las percepciones de los futuros profesores y sus formadores sobre los conocimientos que se están construyendo en el desarrollo del plan de estudios del grado de maestro en educación primaria y la contribución de estos a configurar una identidad profesional determinada. En este proyecto han participado la USC y la UB. Un mismo proyecto en dos contextos universitarios distintos, con planes de estudio diferentes, centrado en un problema de investigación primordial para fundamentar las decisiones curriculares de la formación inicial.

Desde una perspectiva metodológica cualitativa, se realizó un estudio de casos múltiple a partir del análisis de distintos centros universitarios. Los tres casos abordados fueron: la Facultad de Formación del Profesorado en Lugo, la Facultad de Ciencias de la Educación en Santiago de Compostela, con un mismo plan de estudios, ambas pertenecientes a la USC y la Facultad de Educación de la UB. Este artículo está centrado en los resultados procedentes de los dos casos de la USC.

Se emplearon distintas técnicas de recogida de datos y distintas fuentes de información: análisis documental (planos epistemológico, legislativo y curricular); relatos de estudiantes de primero recién ingresados; 
entrevistas en profundidad a profesorado con docencia en el grado, coordinadores de titulación, decanos de las facultades de Santiago y Lugo, estudiantes del segundo curso y tutores de practicum de ambos campus. Se realizaron también dos grupos de discusión con estudiantes de cuarto año de ambos campus -primera promoción de egresados del grado- días antes de finalizar el curso 2013-14.

Para el análisis de los datos se utilizó un modelo mixto de categorización, deductiva-inductiva (Tójar, 2006), que combina el uso de categorías previas procedentes del marco teórico de referencia con la codificación abierta de los datos. Se utilizó como soporte informático el programa Atlas.ti (versión 7) en el que se volcaron las transcripciones realizadas.

El equipo de la USC, estableció dos grandes dimensiones de análisis: conocimiento profesional y formación inicial en el marco del EEES y un conjunto de once macro-categorías, entre las que se encuentra la de la relación teoría-practica. En cada equipo el análisis se llevó a cabo por pares, para reforzar la confiabilidad del proceso. (Véase Martínez Piñeiro, 2017; Montero, Martínez Piñeiro y Colén, 2017, para un análisis minucioso del proceso empírico).

Presentamos resultados procedentes del análisis de la macro-categoría teoría-práctica que recoge cuestiones relacionadas principalmente con la metodología utilizada en la formación y el practicum. No obstante, entendemos la relación entre teoría y práctica transversal al conjunto de los resultados obtenidos en el entramado de las dos grandes dimensiones del estudio, formando parte de la red generada por otras categorías, por ejemplo plan de estudios-estructura-curricular (Gewerc y Alonso-Ferreiro, 2017).

Para identificar la procedencia de las citas incluidas en el texto, en cada una de ellas consta la técnica de recogida de datos empleada [entrevista (Ent.)], [grupo de discusión (GD.)], el sujeto referido [profesorado (Prof.), alumnado (Alum.) o Tutor] y el campus de pertenencia (Santiago -Stgo- o Lugo), y el párrafo del que se extrae la cita.

\section{Resultados y discusión}

El análisis de las representaciones que los formadores -profesores de la facultad y tutores de practicum- y estudiantes tienen respecto a la re- 
Relaciones entre teoría y práctica en la formación inicial. Percepciones de formadores y estudiantes del Grado de Maestro en Educación primaria

Lourdes Montero Mesa

lación entre teoría y práctica en el desarrollo del plan de estudios del Grado de Maestro en Educación Primaria en la USC, evidencia algunas ideas recurrentes que podrían estar orientando y dando cuenta del trabajo que se realiza en las aulas de la formación inicial y en las de los centros educativos para los diferentes tipos de practicum.

Los resultados obtenidos en la macro-categoría teoría-práctica se analizan con el esquema empleado en el desarrollo del marco teórico, evidenciado en el análisis de resultados: por un lado, argumentos potencialmente explicativos de la separación entre ambas y, por otro, reclaman su interrelación y co-implicación.

\subsection{De la separación entre teoría y práctica}

La percepción de la separación entre la teoría, entendida como el desarrollo de las materias del plan de estudios (contenidos y estrategias metodológicas) y la práctica, asimilada a las prácticas en las escuelas, en la organización del plan de estudios es puesta de relieve por estudiantes de cuarto curso de la primera promoción de los estudios del Grado, a punto de convertirse en egresados, con esta irónica y contundente afirmación:

Nuestra titulación separa muy bien la teoría de la práctica, en eso vamos súper bien. Eso sí que está bien. Que separan las materias de lo que es el practicum, el tiempo del practicum es sagrado. (G.D.Stgo.248).

La teoría precede a la práctica y ésta se concibe subordinada a la teoría, como aplicación en el aula, en las de la facultad y en las aulas de los centros de educación primaria y, en consecuencia, se reclama más aplicación para 'resolver problemas'. Para el alumnado, disponer de respuestas a la pregunta 'cómo hacer' salvaría la brecha entre lo que se dice y las expectativas de que sean ellos quienes integren individualmente las diversas propuestas teóricas. Para Martínez Piñeiro (2017), una de las ideas centrales que marcan la percepción del alumnado sobre el conocimiento profesional construido a lo largo del grado es la 'desconexión' entre la teoría y la práctica y la 'escasa' formación práctica recibida. Han adquirido conocimientos teóricos de mayor o menor relevancia, pero no han aprendido a cómo actuar en un aula. 
La sensación es que la formación que han recibido aquí son muchos fundamentos teóricos, que luego no saben aplicar al aula. Porque en el aula la mayor dificultad es resolver los problemas. (Ent.Prof.2.Lugo.130).

(...) quizás es muy pronto, para saberlo porque aún este año es la primera promoción que sale, pero yo creo que se ha construido todo siempre desde la teoría, es decir, nunca desde la experiencia, yo creo que es nuestro problema metodológico en general (Ent.Prof. 5.Lugo.119).

Llevo no sé cuántos años siendo alumna del sistema educativo, no sé cuántos años escuchando la lección magistral del profesor. Llegamos a Magisterio y dicen que no pero lo siguen haciendo. No nos dicen cómo hacer, nos dicen que hay que hacer otra cosa pero no nos dicen cómo hay que hacer otra cosa, entonces ¿cómo hacemos? (Ent.Alum.1.Stgo.578).

Se produce una cierta satisfacción con el nivel de actualización de los contenidos a la vez que se echa en falta una mayor dedicación a diseñar acciones y crear situaciones para su apropiación experiencial, facilitando el desarrollo de capacidades de resolución de problemas complejos. Una cierta queja sobre la hegemonía de la teoría sobre la práctica que contrasta con el testimonio de una tutora de practicum para quien está claro el valor de la teoría y su potencial interrelación con la práctica:

Eu creo que en xeral as informacións e os traballos que se fan no Grao teñen en conta os avances do coñecemento pedagóxico e didáctico, pero, creo que non están ben conectados. É dicir estamos transmitindo unha información moi actualizada, pero non de tal maneira que o alumno se apropie dela. É dicir que queda como unha información verbal e non tanto a experiencia de facer. Por exemplo, podemos estar dando una información moi actualizada acerca do método de proxectos, pero logo nos aquí non creamos ningún proxecto; ou podemos estar falando dos modelos cooperativos, pero logo nos non creamos situacións de aprendizaxe cooperativo. (Ent.Prof.1.Stgo.34).

(...) prevalecen os contidos académicos sobre a construción de competencias que teñen que ver coa posibilidade de mobilizar eses coñecementos para abordar problemas complexos en situacións realistas. (Ent.Prof.1.Stgo.36).

(...) una de las cosas que más me preocupaba siempre como tutora es hacerles ver la importancia que tiene la teoría que estudian en la universidad con la práctica que aplican en la escuela; porque es muy frecuente que los alumnos Ileguen al aula y te dicen: claro, es que ahora estoy aquí y todo lo que estudias en Magisterio no te vale para nada. (Tutor.3.056). 
Relaciones entre teoría y práctica en la formación inicial. Percepciones de formadores y estudiantes del Grado de Maestro en Educación primaria

Lourdes Montero Mesa

La dicotomía entre teoría y práctica está servida en la organización del Plan de Estudios, en la secuencia temporal de las áreas y disciplinas, en la organización de tiempos y espacios, el tamaño excesivo de los grupos y los problemas organizativos del practicum (Álvarez Nuñez, 2017; Gewerc y Alonso Ferreiro, 2017). Cabe preguntarse si otra organización es posible. Como hipótesis de trabajo, saber que una gran parte de los estudiantes egresados no encontrarán un lugar al sol del mundo laboral de la enseñanza, podría estar provocando en las instituciones de formación inicial un efecto de pérdida de relevancia de su proyección social y de desánimo.

Cabe también Ilamar la atención sobre la complejidad de un plan de estudios en el que concurren un número elevado de áreas de conocimiento (más de 20), en unos tiempos y espacios limitados, con concepciones distintas y quizás escasamente compartidas sobre qué significa formar futuros profesores $y$, probablemente, sin las condiciones para hacerlo competentemente.

Un estado de cosas procedente de la historia del currículo de la formación inicial del profesorado de educación primaria, derivado de la visión de lo que un maestro necesita conocer, mantenido en la estructura acordada por la UNESCO, modificada a tenor de las reformas de los currículos de Educación Primaria, con el correlato posterior de la de los planes de estudio de las instituciones de formación. Una estructura curricular predeterminada por las decisiones políticas de la administración educativa en cada momento histórico. La profesión docente es una profesión regulada con las directrices marcadas en las correspondientes órdenes ministeriales. En el plan de estudios del Grado de Maestro en Educación Primaria (Orden ECI/3857/2007), de los 240 créditos ECTS del título, 210 están previamente marcados con posibilidades de redistribución.

Los contenidos escolares de la educación primaria constituyen una de las fuentes que alimentan la toma de decisiones del plan de estudios (una cuestión controvertida para algunos de los formadores de nuestro estudio por los desajustes producidos por esa vinculación), de modo que a más campos del saber intervinientes, más previsible resulta la fragmentación curricular del plan de estudios de la formación inicial lo que amenaza convertirla en un "callejón sin salida". Fragmentación curricular y aumento incesante de demandas, son algunos de los resultados analizados en otros trabajos de difusión del estudio que comentamos (Gewerc y Alonso Ferreiro, 2017; Zapico, Martínez Piñeiro y Montero, 2017). 
La separación entre la teoría y la práctica se percibe también a través de la organización de las clases en "expositivas" ("la teoría se da, se deposita en algún lugar, se lee...") e "interactivas", estrategia organizativa diseñada por la USC para atender el mandato metodológico del EEES de una mayor implicación de los alumnos en sus procesos de aprendizaje:

La expositiva representa casi la mitad de la docencia presencial, creo que tiene demasiado peso, hay que ir más a lo práctico, que el alumno aprenda haciendo. La interactiva, o la parte de lo que llamamos práctica, debería tener una referencia con respecto al aula que, si fuese posible, requiere de una organización un poco compleja. Requiere la colaboración, evidentemente, de los centros de primaria. Pero, muchas de las teorías de las que se parte, supuestos, conceptos, etc. el alumno del grado de maestro, debería verlos allí, en el aula específicamente. (Ent.Prof. 2.Lugo.72).

(...) o ensino sigue estando moi centrado nos contidos escolares, aínda que na organización que se fai nesta universidade, na que a dimensión práctica é a través das clases interactivas, pois iso gañou visibilidade. É dicir, repártese metade e metade a ensinanza que chamamos expositiva, máis centrada na presentación de temas e o que é a aplicación desto. Eu creo que isto é un avance, pero aínda estamos nun camiño de construír unha formación centrada en situacións que teñen que ver co que vai ser logo o traballo de aula. (Ent.Prof.1.Stgo.42).

Cabe salir al paso de una expectativa de reparto en la que las instituciones universitarias se encargan de las teorías y los centros educativos de la aplicación práctica. En ambos espacios de formación se aprenden, explícita e implícitamente, tanto teorías como prácticas, efecto del carácter moldeador del currículo y de la influencia de los respectivos formadores. Distancia e interrelación son dos polos de un continuum en constante movimiento en la mirada de los implicados.

La relación entre la teoría y la práctica no se puede resolver en educación desde la esperanza de disponer de un sistema teórico determinante de la práctica. El sistema educativo

como complejo de personas que trabajan en él, como entramado de relaciones personales, de relaciones con el mundo externo, con la realidad económica, social y cultural, como aparato institucional regido por una cultura propia y por regulaciones externas, no puede entenderse ni gobernarse 
Relaciones entre teoría y práctica en la formación inicial. Percepciones de formadores y estudiantes del Grado de Maestro en Educación primaria

Lourdes Montero Mesa

apoyado en la creencia de que las teorías determinan y gobiernan la realidad.

No cabe este tipo de idealismo, pero tampoco un determinismo en el que a las ideas no les quepa misión alguna en la configuración del mundo". (Gimeno, 1998, p. 33)

Para algunos de los implicados hay materias con más "obligación" de interacción entre teoría y práctica, entre las que se nombra a las didácticas específicas:

... las distintas didácticas, que son las que pesan, deberían tener su dimensión teórica, su dimensión aplicada en el aula de la facultad, pero también deberían tener su reflejo en los centros de primaria. (Ent.Prof.2.Lugo.073).

Esta expectativa encuentra su correlato en las investigaciones de algunos didactas específicos para quienes sus áreas de conocimiento constituyen puentes para construir la relación teoría y práctica (García Pérez, 2015; Porlán y Rivero, 1998; Porlán, Rivero y Solís, 2011). Conocer cómo contribuye esa especificidad a la construcción de conocimiento profesional, comunicarla y compartirla, merece la pena; reclamar, asimismo, un mayor diálogo entre los diversos formadores sobre el conocimiento que sus disciplinas propician.

De las declaraciones de los implicados se educe claramente que hay materias que exigen más de la acción y materias que menos, de suerte que las primeras presentan mayores opciones de relación entre teoría y práctica e incluso, un tratamiento en simultáneo ("no lo hago, las trabajo conjuntamente..."). Emergen cuestiones de interés como la relacionada con la influencia del perfil de cada área en las posibilidades de relación entre teoría y práctica.

Cochran-Smith y Villegas (2016), en su revisión del grupo de estudios -preparing teachers to teach particular subject matters- coinciden en este interés al señalar que,

generally worked from three assumptions that are prevalent in research on teaching and teacher education: 1) To thrive in contemporary society, all school students need opportunities to develop sophisticated cutting-edge content knowledge as well as problem-solving and critical-thinking skills in key subject areas; 2) many aspects of curriculum, teaching and assessment are specific of subject matter areas; and 3) teacher preparation programs should 
provide opportunities for teacher candidates to learn subject matter specific knowledge, ideas, teaching methods, and classroom practices. (p. 466).

Los formadores vehiculan modelos de profesor a través de los contenidos, estrategias y herramientas manejadas, "poniéndose en la piel de las personas", facilitando la vivencia del significado de ser profesor de un área determinada, lamentando en ocasiones el alejamiento de la institución universitaria de la realidad de las escuelas, que el practicum permite, en simultáneo, constatar y concienciar:

Eles van ser e elas, mestres de Educación Física, e teñen que facer clases, teñen que ver como as fago eu. Pero logo cada neno é un mundo, e cada un de nós tamén somos un mundo. E sobre todo o que intento é que entendan que a nosa función é dar ferramentas, e empezar a construír esa pirámide de coñecemento, para que o día que saian e empecen a traballar teñan unha base. (Ent.Prof. 7-Lugo.277).

La relación entre los formadores de los distintos ámbitos institucionales -universidad y centros educativos- que concurren en la formación inicial es, sin duda, un asunto relevante para la relación entre la teoría y la práctica. La búsqueda de una mayor y más provechosa interrelación entre ambas instituciones lleva tiempo configurando el quehacer de formadores e investigadores (por ejemplo, Bolívar, 2006; Gravani, 2008; Vaillant y Marcelo, 2015; Zeichner, 2010). No lo es tanto la investigación sobre las relaciones formadores y tutores que se diluye en las relaciones institucionales. Los testimonios seleccionados ponen sobre la mesa la opinión crítica de los profesionales en ejercicio y la puesta en cuestión de la solvencia de los formadores de la universidad, para quienes se reclama que tengan alguna experiencia del ámbito y nivel para el que preparan a los futuros profesores, una cuestión que comparten los estudiantes. ¿Cómo se transita, en el caso de poseerla, desde la experiencia práctica en la etapa educativa de referencia a la formación del profesorado? ¿Es una cuestión de transferencia o requiere un conocimiento de segundo nivel? (Korthagen, 2016; Zeichner, 2005).

(...) Sempre lles digo, claro ao ser eu mestra, que aparte de toda esa teoría que vas lendo doutros autores, que enriqueceron o meu coñecemento práctico, teño recursos sobre a práctica. (Ent.Prof.7.Lugo.277). 
Relaciones entre teoría y práctica en la formación inicial. Percepciones de formadores y estudiantes del Grado de Maestro en Educación primaria

Lourdes Montero Mesa

Y luego hay otra dimensión que es más seria que todo esto, los profesionales que están en ejercicio, se cansan de repetirnos que la mayor parte de la teoría que defendeís en la escuela, no tiene sentido en el aula, vosotros estáis totalmente fuera de lugar, no sabéis lo que es un aula de primaria. A ver qué sucede ahora pero me temo que la opinión va a seguir siendo esta. (Ent.Prof. 2.Lugo.73).

Los que les dais clases aquí (en la universidad), no sé qué contacto tenéis en realidad con la práctica habitual que tiene un colegio, no sé si estáis un poco ya en otra onda, no lo sé. O sea, es muy distinta la teoría de la práctica, muy distinta, bastante distinta la teoría de la práctica. Entonces ¿que vosotros deberíais saber un poco más lo que hacen? Sí porque sois vosotros los que los estáis formando. Bueno, nosotros también los estamos formando, sí, a lo mejor los estamos deformando. (Tutor1.06)

La diversidad de formadores existentes: unos vienen de la teoría y otros vienen de la práctica, de 'hacer cosas'. Una realidad compleja que, quizás, está en la base de una ausencia de puntos de vista compartidos. Cada formador tiene su punto de vista sobre lo que hay que "dar" -currículo, experiencias, sobre los conocimientos a obtener. Pero ¿con quien lo ha compartido, discutido? No deja de ser curioso que los formadores sean uno de los componentes de la formación inicial menos estudiado, máxime cuando se dispone de evidencias sobre la importancia de su influencia (Murray, 2016; Korthagen, 2016; Zeichner, 2005). A la pregunta sobre cómo perciben la relación entre la teoría y la práctica y como la perciben los alumnos responden:

Bueno, eu creo que claramente predomina a teoría sobre a práctica. E iso tamén ten unha explicación biográfica: moitos dos profesores que imparten clases na Facultade nunca foron profesores de aula de Infantil ou de Primaria, os seus intereses académicos foron por outro lado e tampouco se preocuparon nin de visitalas, ou de estar en contacto con profesores, mestres. Noutros casos, en cambio, son profesores que durante unha parte da súa biografía profesional foron profesores nalgúns dos niveis educativos e por tanto a eles élles máis fácil facer ese engarce, pero eu creo que está lonxe de ser suficiente. Predomina a nosa visión máis académica, máis dos propios estudos da carreira de procedencia. (Ent.Prof.1.Stgo.45-46).

$(, \ldots$ ) en España yo creo que hay un gran problema de una educación que no se ha sabido adaptar, ni en la transición; una metodología obsoleta donde 
lo que falta es experiencia. Entonces yo lo que intento dar a los alumnos son experiencias para que ellos sean críticos y adquieran su propio conocimiento de las cosas. Creo que un maestro es una persona que también tiene que estar comprometida con su entorno. (Ent.Prof.5.Lugo.99).

\subsection{De la co-implicación entre la teoría y la práctica}

La relación entre teoría y práctica se establece en ocasiones de manera sencilla practicando el principio de coherencia:

Me tocó con una tutora [supervisora] que me ha encantado. ¿Por qué? Porque se implicó. Me decía “¿Usted como va? Muy bien, vamos a estar en contacto, cuénteme todas sus experiencias y voy a pasar un día tres horas contigo allí. Explícame todo lo que quieras. Cómo funciona, qué no funciona, cómo te sientes, todo lo que tú quieras". Entonces, eso para mí fue maravilloso, que mi tutor viniera allí, estuviera toda una mañana conmigo, maravilloso. Entonces, eso es integración teoría-práctica, considero yo. (G.D.Stgo.252).

Hay personas que sí, que hacían una clase expositiva, una clase interactiva, más o menos expositiva más o menos interactiva. Pero muchos profesores, bueno algunos por lo menos, derribaban esa barrera, en tanto las sesiones expositivas como las interactivas consistían en desenvolver habilidades, hacer trabajos, en grupo o no, hacer debates, adquirir competencias, habilidades, actitudes e información de un xeito que non era 'ponte y copia'. Para mí eso es lo mejor. (G.D.Stgo.449)

La preocupación por una mayor relación entre teoría y práctica está presente en los distintos colectivos implicados que, en algún caso, advierten del exceso de expectativas puestas en el practicum para dar respuesta a las interacciones entre las teorías trabajadas en la universidad, las prácticas observadas en las aulas universitarias y en los centros escolares, y el aprendizaje de las complejas relaciones entre ambas, marcando la responsabilidad para ello de los propios formadores.

(...) Pero muchas de las teorías de las que se parte, supuestos, conceptos, etc., el alumno del grado de maestro debería verlos allí en el aula específicamente, que quizás luego el practicum no le va a dar lugar a ello, porque el practicum es un practicum que abarca todo y como abarca todo, supongo que lo que le quedará al final será una visión general del centro, del aula, de 
Relaciones entre teoría y práctica en la formación inicial. Percepciones de formadores y estudiantes del Grado de Maestro en Educación primaria

Lourdes Montero Mesa

los problemas de aula etc. Y hay muchos matices, porque el aprendizaje es mucho más rico que todo eso. (Ent.Prof.2.Lugo.072).

La comprensión del practicum como un elemento nuclear en la formación inicial, con un aumento sustantivo del número de créditos en el grado en comparación con la diplomatura, hace de él un elemento central para las diversas asignaturas. Más allá del compromiso de los supervisores de practicum, los formadores tendrían la obligación de establecer una mayor relación con él antes, durante y después (White y Forgasz, 2016; Zeichner, 2010). Algo que se produce:

(...) pero yo tengo la percepción de que en planes de estudio anteriores la conexión del resto de las materias con respecto a lo que entonces se llamaba prácticas escolares, era mínima, hacían sus prácticas y volvían. Yo creo que ahora en este plan de estudios dado que el practicum ocupa mucho más, hay mucha más relación con el resto de las materias, se aprovecha mucho más lo que se hizo en el practicum para trabajar las materias. (Ent.Prof.1.Lugo.110).

Para ir más allá de descansar en el practicum como el espacio de encuentro de prácticas y teorías, se necesitan mecanismos de intermediación. Entre otros, la construcción de "pequeñas pedagogías" (Rozada, 2006); los "espacios híbridos" (Zeichner, 2010); los profesores como investigadores (Stenhouse, 1984; Elliott, 2010); el enfoque realista (Korthagen, 2010); la estrategia IT-INSET, de configuración de equipos mixtos de formadores, futuros profesores y tutores (Montero, Requejo, Vez y Zabalza, 1990); las lessons study (Pérez Gómez, Soto y Serván, 2015) la indagación de los "saberes experienciales" y su uso en propuestas de investigación y formación (Blanco, Orozco y Arbiol, 2016³; Contreras, 2013, 2016).

La necesidad de interrelación teoría y práctica, práctica y teoría, no debiera pretender convertirse en un recetario pero sí en pistas para resolver las preguntas que inquietan a los estudiantes. La teoría es esencial para transformar la realidad pero se necesita para ello la concurrencia entre la teoría y la práctica porque si no "la realidad nos va comiendo", pero esa relación imprescindible "hace agua":

2 Véase el monográfico dedicado por la Revista Interuniversitaria de Formación del Profesorado (RIFOP), 2015, número 84.

3 Cuadernos de Pedagogía 470, septiembre 2016, ha dedicado a este tema la sección "tema del mes". 
(...) La relación entre lo que ellos esperarían de la formación en una facultad, que no fuese un recetario, no puede serlo evidentemente, pero sí que sirviese de base para resolver los problemas diarios de aula. Y ¿qué hago con un alumno indisciplinado? ¿Cómo mantengo el orden en un aula, en un grupo, para que se produzca el aprendizaje? Y ¿cómo hago con un alumno que no logra alcanzar los niveles? Y con los padres que no asisten a las reuniones que convoco (Ent.Prof.2.Lugo.136).

(...) teoría, práctica, realidad. O sea, la realidad modela la práctica y la práctica modela la realidad. Si nosotros tenemos teoría y después no tenemos práctica, no seremos capaces de cambiar la realidad. Y si nosotros tenemos realidad pero no tenemos teoría, no seremos capaces tampoco de cambiar esa realidad, sino que la realidad nos va comiendo. (Ent.Prof.3.Lugo.108).

(...) Lo que no se puede hacer es disociar los contenidos de la práctica. Un poco lo que estamos diciendo aquí es que para mí esto es un poco como la escuela de peluquería, en el sentido de que tú vas a clase pero haces prácticas, o sea, practicas. Entonces aquí damos sociología, psicología, después, es todo teoría. No nos enfrentamos realmente, no vemos eso en la realidad. Debería estar más unificada la teoría y la práctica. Pero si lo ves realmente en la práctica, ahí es cuando realmente dices: si esto es así (G.D.Lugo.044).

Los tutores de los centros son modelos de referencia, más poderosos que los formadores de la universidad debido, hipótesis de trabajo, a la legitimidad que les otorga el contexto social de su práctica. Los testimonios que siguen son expresivos tanto de la impronta que dejan sus actuaciones como modelos de acción cuanto de la defensa de la profesionalidad de los maestros de primaria:

(...) si tú ahora tuvieras ya que ponerte a dar clases ¿cómo las darías? Dijo, bueno, pues mira las daría más o menos como haces tú. Dije, pero ¿no habría nada que me pudieras a mí aportar de lo que tuviste en el otro colegio, con el otro tutor o tutora? No sé, dijo, no, yo las daría más o menos como tú. Si fuera un colegio pequeño pues a lo mejor trabajaría en proyectos. (Tutor.4.144).

(...) una buena práctica tiene que estar sustentada en una buena teoría yo creo que uno de los errores grandes que hay con respecto a la escuela, a la carrera de magisterio, es pensar que nosotros somos cuidadores de niños y nosotros somos profesionales de la educación. Entonces tenemos un ámbito profesional que tiene una parte científica que tiene que estar sustentada en 
Relaciones entre teoría y práctica en la formación inicial. Percepciones de formadores y estudiantes del Grado de Maestro en Educación primaria

Lourdes Montero Mesa

una teoría, mira, el fundamento es éste. Que relacionen los dos mundos

(Tutor.3.060).

Cabe preguntarse por el tremendo galimatías multidisciplinar para la construcción del conocimiento profesional docente por el profesorado del futuro ante la enorme complejidad de los significados de ser maestro en este siglo XXI. La relación entre la teoría y la práctica continúa siendo una cuestión inquietante.

\section{Conclusiones}

La riqueza de las percepciones de formadores, tutores y futuros profesores, desborda los límites de este artículo que muestra unas pinceladas de un retrato inacabado donde se ponen de manifiesto las tensiones entre teoría y práctica en el ámbito de la formación inicial. El análisis e interpretación de los datos evidencia un modelo de separación teoría y práctica en el que la teoría precede a la práctica y ésta es comprendida como aplicación de la teoría. Una situación que genera tensiones e insatisfacción entre formadores y estudiantes, pero también preocupación y la búsqueda de estrategias alternativas para la co-implicación deseable entre ambos tipos de saberes. Sin embargo, los obstáculos y ataduras para hacerlo parecen ser más poderosas que los deseos de cambiar una realidad socialmente construida en la que el juego dialéctico, bidireccional, se constituya en principio para la acción. La complejidad de la formación inicial del profesorado de primaria, la diversidad de saberes, personas y contextos presentes, no parece reconocerse en toda su dimensión y merecer, social y políticamente, lo que otros ámbitos de conocimiento y acción ya poseen.

A la luz de las informaciones obtenidas sobre la relación teoría y práctica planteamos como hipótesis emergente la desconexión en el desarrollo del plan de estudios de la formación inicial entre profesorado, estudiantes y tutores, colectivos directamente implicados en su desarrollo.

No resulta fácil afrontar el doble mandato de transmisión del saber de las disciplinas presentes en los planes y programas y ayudar a configurar saberes y compromisos profesionales. La actividad profesional de la enseñanza, es una actividad eminentemente práctica que sólo puede 
transformarse a partir de la comprensión de los esquemas teóricos que la guían. Los conocimientos de los docentes "no son una suma de 'saberes' o de 'competencias' que uno podría describir y encerrar en un libro o un catálogo de competencias. Se trata de saberes integrados a las prácticas docentes cotidianas, las cuales están ampliamente condicionadas por intereses normativos, cuando no éticos y políticos" (Tardiff, 2013, p.39).

\section{Referencias bibliográficas}

Álvarez Álvarez, C. (2011). La relación teoría-práctica en la enseñanza y el desarroIlo profesional docente. Un estudio de caso en Primaria. Tesis Doctoral. Departamento de Ciencias de la Educación. Universidad de Oviedo (http://hdl.handle. net/10651/13012).

Álvarez Nuñez, Q. (2017). La dimensión olvidada. El papel de los factores organizativos en la formación de los Maestros de Primaria según las percepciones de su profesorado. Profesorado. Revista de Curriculum y Formación del Profesorado, 21 (1), 164-181.

Ben-Peretz, M. (2011). Teacher knowledge: What is it? How do we uncover it? What are its implications for schooling? Teaching and Teacher Education, 27, 3-9.

Blanco, N., Orozco, S. y Arbiol, C. (2016). Un saber para la formación: aprender con docentes. Cuadernos de Pedagogía, 470, 46-48.

Bolívar, A. (2006). La formación inicial del profesorado y el desarrollo de las instituciones de formación. En J. M. Escudero y A. Luis (Eds.). La formación del profesorado y la mejora de la educación (pp.123-154). Barcelona: Octaedro.

Britzman, D. P. (1986). Cultural myths in the making of a teacher: biography and social structures in teacher education. Harvard Educational Review, 56 (4), 442-472.

Carr, W. (1990). Hacia una ciencia crítica de la educación. Barcelona: Laertes.

Carr, W. Y Kemmis, S. (1988). Teoría crítica de la enseñanza. La investigación-acción en la formación del profesorado. Barcelona: Martínez Roca.

Cochran-Smith, M., \& Villegas, A. (2016). Research on Teacher Preparation: Charting the Landscape of a Sprawling Field. In Gitomer, D., \& Bell, C. (Eds.). Handbook of Research on Teaching. 5th Edition (pp. 439-547). Washington DC: American Educational Research Association.

Colén, $M^{a}$. T. (coord. y ed.) (2017). Retos y certezas sobre la construcción del conocimiento práctico en la formación de maestros. Una visión caleidoscópica. Barcelona: Octaedro-ICE-UB.

Contreras, J. (2013). El saber de la experiencia en la formación inicial del profesorado. Revista Interuniversitaria de Formación del profesorado, 78 (27, 3), 125-136.

Contreras, J. (comp.) (2016). Tensiones fructíferas: explorando el saber pedagógico en la formación del profesorado. Una mirada desde la experiencia. Barcelona: Octaedro.

Elliott, J. (2010). El "estudio de la enseñanza y el aprendizaje": una forma globalizadora de investigación del profesorado. Revista Interuniversitaria de Formación del profesorado, $68(24,2), 227-242$. 
Relaciones entre teoría y práctica en la formación inicial. Percepciones de formadores y estudiantes del Grado de Maestro en Educación primaria

Lourdes Montero Mesa

Escudero, J. M. (1993). La construcción problemática de los contenidos de la formación del profesorado. En L. Montero y J. M. Vez (Eds.). Las Didácticas Específicas en la Formación del Profesorado (I) (pp. 71-91). Santiago de Compostela: Tórculo.

Escudero, J. M. (2006). La formación del profesorado y la garantía de una buena educación para todos. En J.M. Escudero y A. Luis (Eds.). La formación del profesorado y la mejora de la educación (pp. 21-51). Barcelona: Octaedro

Escudero, J. M. Y Luis, A. (Eds.) (2006). La formación del profesorado y la mejora de la educación. Barcelona: Octaedro

Ferreres, V. (1998). ¿Y qué pasa con los tutores de practicas en la Universidad? ¿Cómo se forman? ¿Qué papel?. Qué funciones? ¿Qué formación?. En M. A. Zabalza (Ed.). Los tutores en el practicum. Funciones, formación, compromiso institucional (pp.129148). Pontevedra: Diputación Provincial.

Fullan, M. (2002). Los nuevos significados del cambio en educación. Barcelona: Octaedro.

García Pérez, F. F. (2015). El conocimiento escolar en el centro del debate didáctico. Reflexiones desde la perspectiva docente. Con-Ciencia Social, 19, 49-62.

Gewerc, A. Y Alonso-Ferreiro, A. (2017). Influencias del plan de estudios del Grado de Maestro de Primaria en el conocimiento profesional del profesorado. El caso de la Universidad de Santiago de Compostela. Profesorado. Revista de Curriculum y Formación del Profesorado, 21 (1), 39-58.

Gimeno, J. (1998). Poderes inestables en educación. Madrid: Morata.

Gravani, M. (2008). Academic and practitioners: partners in generating knowledge or citizens of two diferent worlds? Teaching and Teacher Education, 24, 649-659.

Hargreaves, A. (1999). Hacia una geografía social de la profesión docente. En A. Pérez Gómez, J. Barquín y J. F. Angulo (Eds.). Desarrollo profesional del docente: Política, investigación y práctica (pp.119-145). Madrid: Akal.

Khortagen, F. (2010). La práctica, la teoría y la persona en la formación del profesorado. Revista Interuniversitaria de Formación del Profesorado, 68 (24, 2), 83-101.

Khortagen, F. (2016). Pedagogy of Teacher Education. . In J. Loughran \& M. L. Hamilton (Eds.) International Handbook of Teacher Education. Vol. 1 (pp. 311-70). Singapore: Springer Science+Business Media.

Labaree, D. F. (1999). Poder, conocimiento y racionalización de la enseñanza: genealogía del movimiento por la profesionalidad docente. En A. Pérez Gómez, J. Barquín y J. F. Angulo (Eds.). Desarrollo profesional del docente: Política, investigación y práctica. (pp. 16-51). Madrid: Akal.

Lieberman, A. Y Miller, L. (2003). La indagación como base de la formación del profesorado y la mejora de la educación. Barcelona: Octaedro

Martínez Piñeiro, E. (2017). Desarrollo del conocimiento profesional a través del plan de estudios del Grado de Maestro. El caso de la Universidad de Santiago de Compostela. En Ma. T. Colén (coord. y ed.) (2017). Retos y certezas sobre la construcción del conocimiento práctico en la formación de maestros. Una visión caleidoscópica (pp. 77-91). Barcelona: Octaedro-ICE-UB.

Medina Moya, J. L. (2017). La relación entre teoría y práctica en la construcción del conocimiento profesional docente: aspectos epistemológicos. En M ${ }^{a}$. T. Colén (Coord. 
Relaciones entre teoría y práctica en la formación inicial. Percepciones de formadores y estudiantes del Grado de Maestro en Educación primaria

Lourdes Montero Mesa

y Ed.) (2017). Retos y certezas sobre la construcción del conocimiento práctico en la formación de maestros. Una visión caleidoscópica (pp. 23-36). Barcelona: OctaedroICE-UB.

Montero, L. (2001). La construcción del conocimiento profesional docente. Rosario: Homo Sapiens.

Montero, L. (2006). Los caminos de la memoria. Reflexiones acerca de la teoría, la práctica, las prácticas. Revista de Ciències de l'Educació, marc, 133-160.

Montero, L. (2008). El retorno del interés por la formación inicial del profesorado. Monográficos Escuela, octubre, 4-6.

Montero, L. (2016). La formación del profesorado: cuestiones perdurables en escenarios de incertidumbre. In C. Mesquita, M. V. Pires \& R. P. Lopes (Eds.). Livro de Atas do 1. ${ }^{\circ}$ Encontro Internacional de Formação na Docência. INCTE (pp. 11-31). Bragança: Instituto Politécnico de Bragança.

Montero, L. (2017). La relación entre la teoría y la práctica en la formación del profesorado. En Ma. T. Colén Riau (coord. y ed.). Retos y certezas sobre la construcción del conocimiento práctico en la formación de maestros. Una visión calidoscópica (pp. 60-76). Barcelona: Octaedro/ICE-UB.

Montero L., Martínez Piñeiro, E. Y Colén, Ma. T. (Eds.) (2017). Los estudios de Grado en la formación inicial de Maestros en Educación Primaria. Miradas de formadores y futuros maestros. Profesorado. Revista de Curriculum y Formación del Profesorado, 21 (1), 1-16. Monográfico: Desarrollo del conocimiento profesional en la formación inicial de maestros.

Montero L., Requejo, Ma. J., Vez, J. M. Y Zabalza, M. (1990). IT-INSET Galicia. Una experiencia de formación centrada en la escuela. En J. López Yañez y B. Bermejo (Coords.). El centro educativo. Nuevas perspectivas organizativa, (47-56). Sevilla: Universidad de Sevilla/GID.

Murray, J. (2016). Beginning Teachers Educators: Working in Higher Education and Schools. In J. Loughran \& M. L. Hamilton (Eds.) International Handbook of Teacher Education. Vol. 2 (pp. 35-70). Singapore: Springer Science+Business Media.

Pérez Gómez, A. I. (1993). La interacción teoría-práctica en la formación del docente. En L. Montero y J. M. Vez (Eds.). Las Didácticas Específicas en la Formación del Profesorado (I) (pp. 29-51). Santiago de Compostela: Tórculo.

Pérez Gómez, A. I., Soto, E. Y Serván, Ma . J. (2015). Lessons Studies: re-pensar y re-crear el conocimiento práctico en cooperación. Revista Interuniversitaria de Formación del Profesorado, 84 (29.3), 81-101.

Porlán, R. y Rivero, A. (1998). El conocimiento de los profesores. Sevilla: Diada.

Porlán, R., Rivero, A. Y Solís, E. (2011). Un modelo de formación para el cambio del profesorado de ciencias. En Primeras Jornadas de Innovación Docente de la facultad de Ciencias de la Educación de la Universidad de Sevilla (pp. 1-9). Recuperado de https://idus.us.es/xmlui/handle/11441/25491.

Prats, E. (2016). La formación inicial docente entre profesionalismo y vías alternativas: mirada internacional. Bordón, 68 (2), 19-33.

Rodríguez Romero, Ma del M. (2001). Las representaciones del cambio educativo. Revista de Educación, 326, 167-184. 
Relaciones entre teoría y práctica en la formación inicial. Percepciones de formadores y estudiantes del Grado de Maestro en Educación primaria

Lourdes Montero Mesa

Rozada, J. Ma . (2006). La formación permanente del profesorado y el desarrollo de una "pequeña pedagogía" crítica. Notas autobiográficas de una vida profesional en la frontera. En J. M. Escudero y A. Luis (Eds.). La formación del profesorado y la mejora de la educación (pp. 197-229). Barcelona: Octaedro

Schön, D. (1992). La formación de profesionales reflexivos. Hacia un nuevo diseño de la enseñanza y el aprendizaje en las profesiones. Madrid: Paidós/MEC.

Stenhouse, L. (1984). Investigación y desarrollo del curriculum. Madrid: Morata.

Tardiff, M. (2004). Los saberes del docente y su desarrollo profesional. Madrid: Narcea.

Tardiff, M. (2013). ). El oficio docente en la actualidad. Perspectivas internacionales y desafíos a futuro. En M. Poggi (Coord.). Políticas docentes: formación, trabajo y desarrollo profesional, (pp. 19-44). Buenos Aires: Instituto Internacional de Planeamiento de la Educación IIPE-Unesco.

Tiana, A. (2013). Los cambios recientes en la formación inicial del profesorado en España: una reforma incompleta. Revista Española de Educación Comparada, 22, 39-58.

Tojar, J. C. (2006). Investigación cualitativa. Comprender y actuar. Madrid: La Muralla.

Vaillant, D. Y Marcelo, C. (2015). El ABC y D de la Formación Docente. Madrid: Narcea.

Veenman, S. (1984). Perceived problems of beginning teachers. Review of Educational Research, 54 (29), 143-177.

White, S. Y Forgasz, R. (2016). The Practicum: The Place of Experience? In J. Loughran \& M. L. Hamilton (Eds.). International Handbook of Teacher Education. Vol. 1. (pp. 231266). Singapore: Springer Science+Business Media.

Zapico, Ma . H, Martínez Piñeiro, EY Montero, L. (2017). Demandas sociales y formación inicial del profesorado: ¿Un callejón sin salida? Profesorado. Revista de Curriculum y Formación del Profesorado, 21 (1), 80-102.

Zeichner, K. (2005). Becoming a teacher educator: A personal perspective. Teaching \& Teacher Education, 21, 117-124.

Zeichner, K. (2010). Nuevas epistemologías en formación del profesorado. Repensando las conexiones entre las asignaturas del campus y las experiencias de prácticas en la formación del profesorado en la universidad. Revista Interuniversitaria de Formación del profesorado, 68 (24, 2), 123-149. 